\section{Materiales de restauración y su aplicación por parte de estudiantes de odontología}

\author{
Restoration materials and its application by students of \\ dentistry
}

\section{Materiais de restauração e sua aplicação por estudantes de odontologia}

\author{
Ana Fernández Goncalvez \\ anamfg24@gmail.com \\ Carla Córdova Perdomo \\ carlacordova07@gmail.com
}

\begin{abstract}
RESUMEN
Introducción: La formación académica es un pilar fundamental en cualquier carrera donde los conocimientos adquiridos representan en conjunto con las habilidades y destrezas, la actitud profesional para llevar a cabo un procedimiento de manera eficiente y eficaz. El conocimiento científico se adquiere mediante el estudio de los fundamentos teórico prácticos de la disciplina con el objeto de proporcionar la mejor atención al usuario o paciente de los servicios odontológicos en los centros de salud. Objetivo: La finalidad de esta investigación fue determinar la relación entre el conocimiento sobre materiales dentales y su aplicación por parte de los estudiantes de la clínica integral I de la Universidad José Antonio Páez en el periodo septiembre- diciembre 2018. Materiales y Métodos: Investigación descriptiva con un diseño de campo, no experimental, transversal. La recopilación de información se realizó mediante un cuestionario y una lista de cotejo. La población estuvo constituida 133 estudiantes de odontología, mediante muestreo de tipo probabilístico se obtuvo la muestra de 44 estudiantes, se aplicó un cuestionario y una guía de observación para correlacionar las variables, mediante un análisis estadístico de Pearson. Resultados y Discusión: Se registró una correlación de tipo imperfecta o relación inversa según Pearson. Conclusión: Se llegó a la conclusión que existe una mediana relación, debido a un gran porcentaje conoce las definiciones, pero no las aplica, lo que expresa la necesidad de reforzar el cumplimiento de los protocolos clínicos para la práctica odontológica.
\end{abstract}

Palabras clave: educación en odontología; métodos; procedimiento; materiales dentales

\begin{abstract}
Introduction: Academic training is a fundamental pillar in any career, the knowledge acquired together with the skills and abilities, the professional attitude to carry out a procedure efficiently and effectively. Scientific knowledge is acquired by studying the practical theoretical foundations of the discipline in order to provide the best care to the user or patient of dental services in health centers. Objective: The purpose of this research was to determine the relationship between knowledge about dental materials and their application by the students of the integral clinic I of the José Antonio Páez University in the period September-December 2018. Materials and Methods: Descriptive research with a field design, not experimental, transversal. The information was collected through a questionnaire and a checklist. The population was constituted 133 students of dentistry, by means of sampling of probabilistic type the sample of 44 students was obtained, a questionnaire and an observation guide were applied to correlate the variables, by means of a statistical analysis of Pearson. Results and Discussion: An imperfect type correlation or inverse relationship according to Pearson was recorded. Conclusion: It was concluded that there is a medium relationship, since a large percentage knows the definitions, but does not apply them, which expresses the need to reinforce compliance with clinical protocols for dental practice.
\end{abstract}

AF: Universidad José Antonio Páez. Venezuela.

CC: Universidad José Antonio Páez. Venezuela.

Key words: dental education; methods; procedure; dental materials. 
AF: Universidad José Antonio Páez. Venezuela.

CC: Universidad José Antonio Páez. Venezuela.

\section{RESUMO}

Introdução: A formação acadêmica é um pilar fundamental em qualquer carreira, o conhecimento adquirido juntamente com as habilidades e habilidades, a atitude profissional para realizar um procedimento de forma eficiente e eficaz. O conhecimento científico é adquirido através do estudo dos fundamentos teóricos práticos da disciplina, a fim de prestar o melhor atendimento ao usuário ou paciente dos serviços odontológicos nos centros de saúde. Objetivo: $O$ objetivo desta pesquisa foi determinar a relação entre o conhecimento sobre materiais odontológicos e sua aplicação pelos estudantes da clínica integral I da Universidade José Antonio Páez, no período de setembro a dezembro de 2018. Materiais e Métodos: Pesquisa descritiva com delineamento de campo, não experimental, transversal. As informações foram coletadas através de um questionário e uma lista de verificação. A população foi constituída por 133 estudantes de odontologia, por meio de amostragem do tipo probabilístico, foi obtida a amostra de 44 alunos, aplicado um questionário e um guia de observação para correlação das variáveis, por meio de uma análise estatística de Pearson. Resultados: Foi registrada uma correlação do tipo imperfeito ou relação inversa de acordo com Pearson. Conclusão: Concluiu-se que existe uma relação média, pois grande parte conhece as definições, mas não as aplica, o que expressa a necessidade de reforçar o cumprimento dos protocolos clínicos para a prática odontológica.

Palavras-chave: educação odontológica; métodos; procedimento; materiais odontológicos

\section{INTRODUCCIÓN}

$\mathrm{L}$ a odontología posee un pasado histórico que se remonta a 3.500 años a.C, ya para esa época se trataban las afecciones dentales que producían dolor y/o infección, con materiales como arena, miel, arcilla, piedras y metales preciosos, entre otros. De ésta manera se puede considerar que se inició el estudio de los materiales, que podrían ser utilizados en la cavidad oral mediante la práctica empírica donde se determinaba cuál de ellos resultaba ser más eficaz.

La odontología como ciencia tiene el objeto de fomentar la salud bucal, diagnosticar, prevenir y curar las enfermedades del aparato estomatognático, para recuperar en el paciente la estética y función. Para llevar a cabo estas funciones el odontólogo debe poseer conocimiento científico de las enfermedades y alteraciones bucales, así como dominar las técnicas del tratamiento, desde su ejecución, conocimiento del instrumental, hasta los materiales con los cuales se trabaja.

En el estudio de la odontología clínica es relevante conocer sobre los materiales dentales, su composición, propiedades físicas, químicas y mecánicas, su interacción en el medio bucal y posibles reacciones del material, todo esto se relaciona con el diagnóstico del clínico y la elección del tratamiento, como consecuencia, se evalúa cuál sería el material dental idóneo para la restauración dental o cualquier otro procedimiento. Debido a las características que deben poseer estos materiales los cuales deben ser compatibles e inocuos a los tejidos, se introduce el término biomaterial $(1,2)$.

La formación dentro de una escuela de odontología, incluye el desarrollo de competencias en ciencias básicas como la biología, física y química, otorgándole al estudiante las bases sobre las patologías bucales y sus tratamientos. Por esto, el conocimiento de los materiales con los cuales los estudiantes trabajarán, debe ser prioridad en el proceso de formación académica.

El desconocer las propiedades y reacciones de los biomateriales, puede ocasionar una inadecuada manipulación que pudiera provocar algún comportamiento adverso del material con 
el consiguiente fracaso del tratamiento odontológico $(3,4)$.

Esta investigación se realizó con el fin de determinar la relación entre el conocimiento sobre materiales dentales y su aplicación por parte de los estudiantes de la clínica integral I de la Universidad José Antonio Páez.

\section{MATERIALES Y MÉTODOS}

I nvestigación descriptiva con un diseño de campo, no experimental, transversal. La recopilación de información se realizó mediante un cuestionario y una lista de cotejo. La población estuvo constituida por todos los estudiantes de odontología cursantes de la asignatura Clínica Integral I de la Universidad José Antonio Páez, 133 sujetos, que representaron el $100 \%$ de la población en estudio. Mediante muestreo de tipo probabilístico se seleccionaron cuarenta (40) alumnos al azar, entre la lista total de estudiantes. Los datos fueron vaciados en una tabla en Microsoft Excel, para luego ser presentados a partir de las técnicas estadísticas descriptivas univariadas y bivariadas a través de tablas de asociación y de medias.

\section{RESULTADOS}

7 osterior a la recolección de datos, se expone a continuación los resultados alcanzados:

Tabla 1. Correlación de variables

\begin{tabular}{llcc}
\hline & \multicolumn{1}{c}{$\begin{array}{c}\text { Conocimiento/ } \\
\text { materiales de } \\
\text { restauración }\end{array}$} & Aplicación \\
\hline $\begin{array}{l}\text { Conocimiento sobre los } \\
\text { materiales de } \\
\text { restauración }\end{array}$ & $\begin{array}{l}\text { Correlación de } \\
\text { Pearson } \\
\text { Sig. (bilateral) }\end{array}$ & 1 &, $007\left(^{* *}\right)$ \\
$\begin{array}{l}\text { Aplicación que se da en } \\
\text { los protocolos } \\
\text { clínicos }\end{array}$ & $\begin{array}{l}\text { Correlación de } \\
\text { Pearson } \\
\text { Sig. (bilateral) }\end{array}$ &, $507\left(^{* *}\right)$ & 1 \\
\hline
\end{tabular}

** La correlación es significativa al nivel 0,01 (bilateral) a $\mathrm{N}$ por lista $=40$

El coeficiente de correlación de Pearson (r) se mide en una escala de 0 a 1 , tanto en dirección positiva como negativa. Un valor de "0" indica que no hay relación lineal entre las variables. Un valor de " 1 " o “-1" indica, respectivamente, una correlación positiva perfecta o negativa perfecta entre dos variables. De acuerdo con los resultados obtenidos, se puede apreciar que la correlación de Pearson en cuanto al conocimiento sobre los materiales de restauración es de 001, lo que quiere decir que hay una mediana relación. 


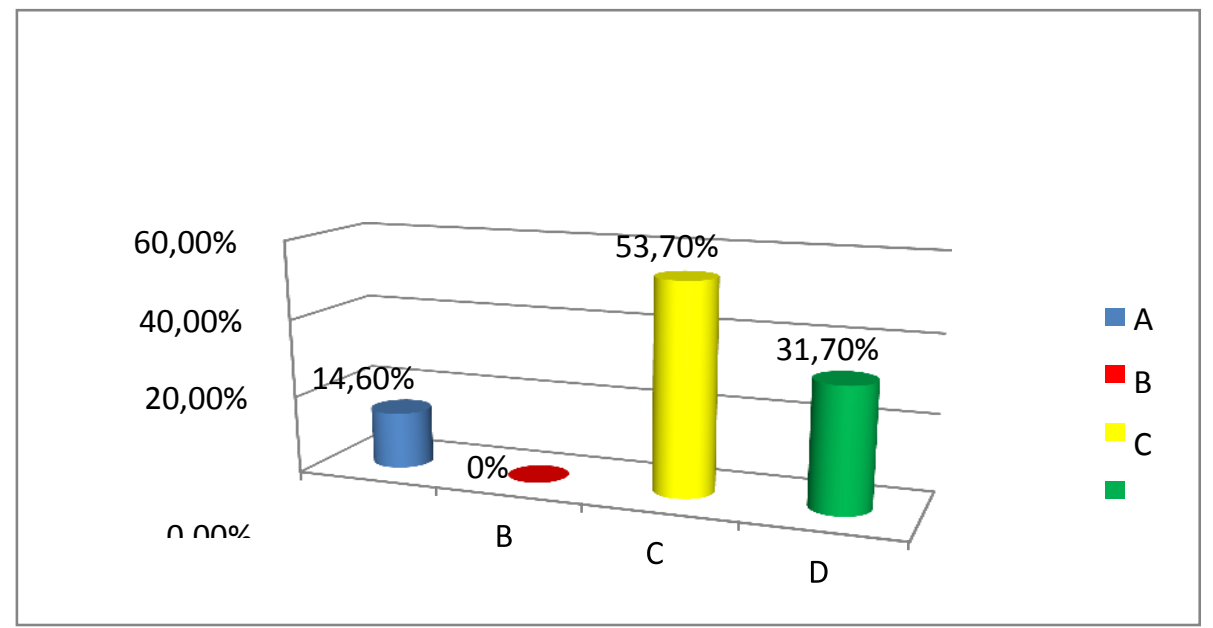

Gráfico 1. Concepto de material de restauración

En el gráfico 1, los resultados evidencian que la mayoría de respuestas emitidas por los sujetos están dispersas, demostrando que tienen mediano conocimiento sobre el concepto de material de restauración, tal cual lo evidencia el coeficiente de correlación.

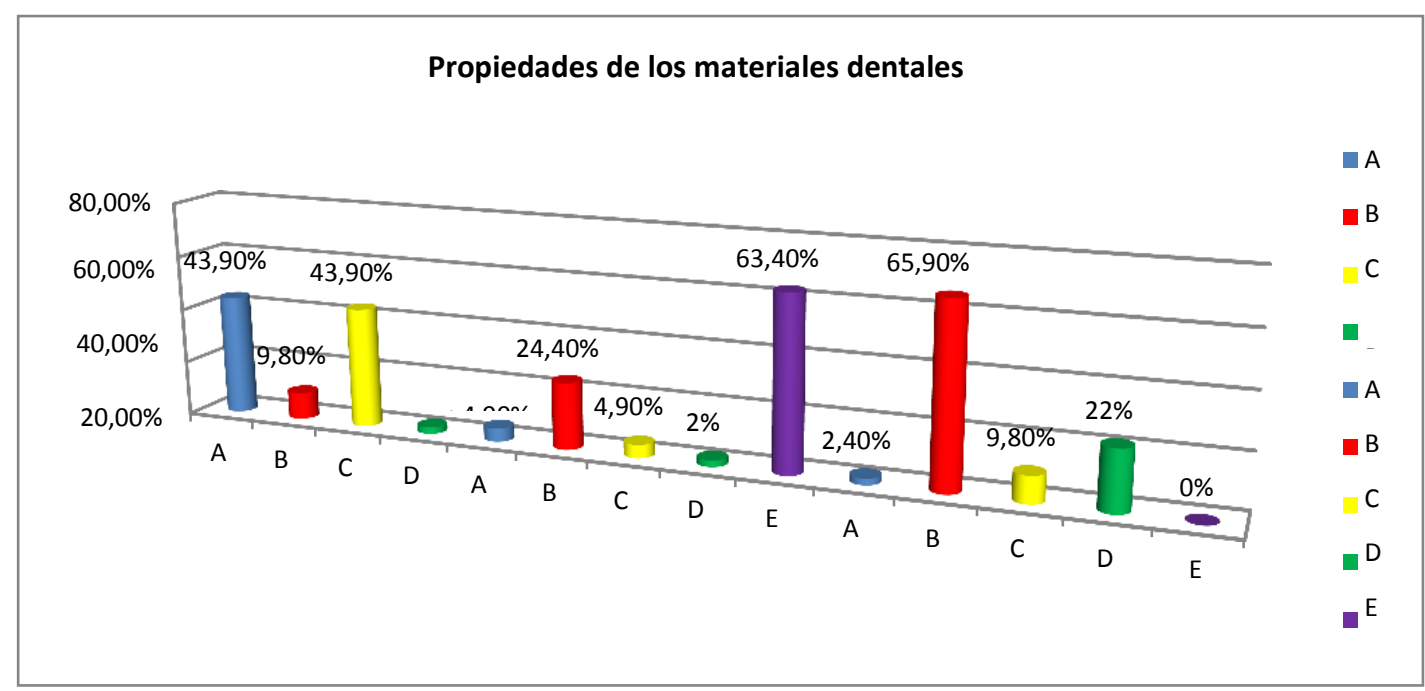

Gráfico 2. Propiedades de los materiales dentales

Dichos resultados permiten deducir que solo una parte de los sujetos encuestados acertaron en la opción, solo un grupo minoritario conocen las propiedades de los materiales dentales. 


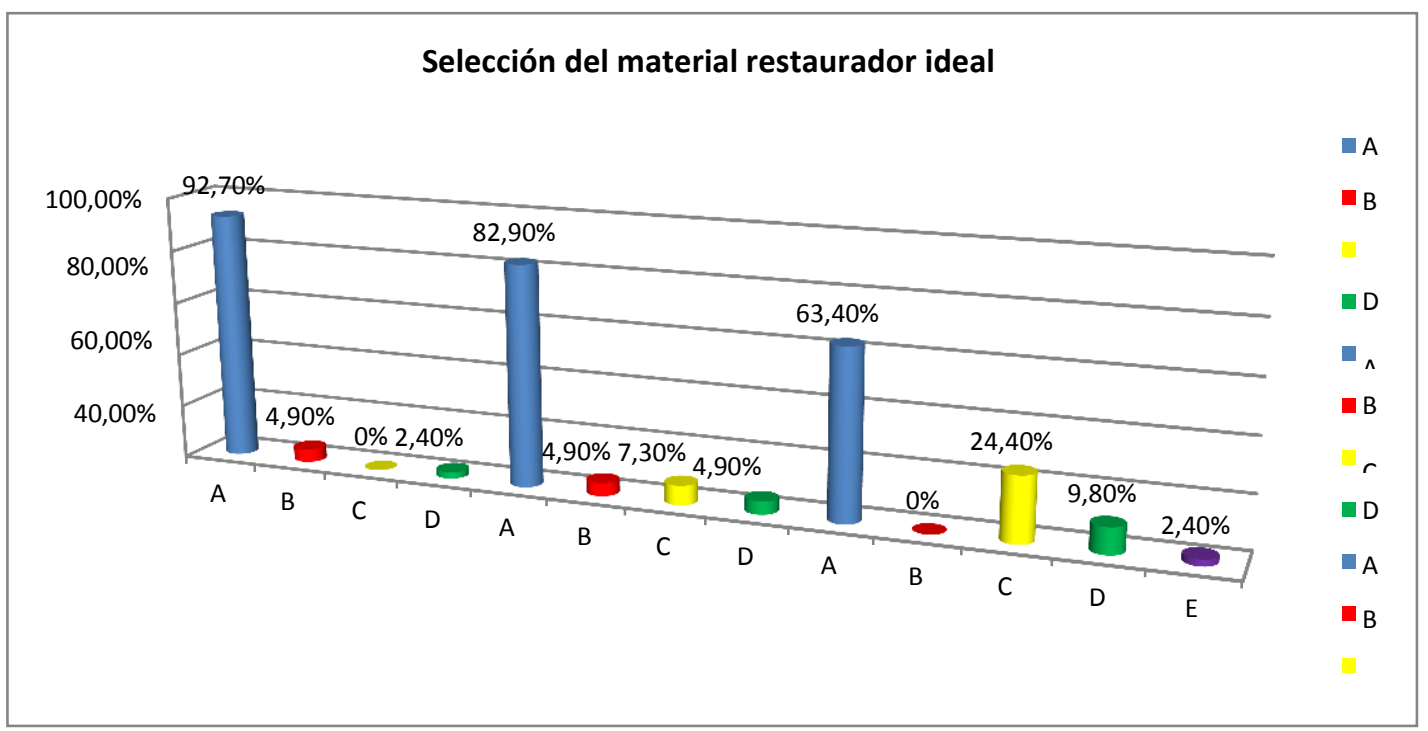

Gráfico 3. Selección del material restaurador ideal

Los resultados indican la mediana relación que existe entre el conocimiento sobre los materiales de restauración y su aplicación por parte de los estudiantes de la Clínica Integral I.

\section{DISCUSIÓN}

$\mathrm{L}$ a odontología es una disciplina dedicada al cuidado de la salud que requiere además de una sólida formación teórica, el desarrollo de habilidades y destrezas procedimentales así como rasgos actitudinales para lograr sus objetivos. Esta actitud resulta reforzada por la constante irrupción de nuevos materiales, el incesante desarrollo de novedosas técnicas y la permanente gestación de procedimientos operatorios revolucionarios. Los diferentes materiales que se usan para tratamientos odontológicos precisan de propiedades beneficiosas que admitan su uso en seres humanos, como son: la de ser inocuos, no tóxicos, no irritantes de los tejidos y deben ser biocompatibles (2).

Existe la posibilidad que cualquiera de las propiedades inocuas estén ausentes de los materiales dentales bien por dificultades durante la manipulación, donde no se tomen las precauciones de protección, o la presencia de algún elemento que en su composición pueda considerarse potencialmente nocivo, o por alguna característica inherente al personal dental o paciente que lo haga susceptible o vulnerable (3). Estas posibilidades de riesgo pueden presentarse aún con los materiales más comúnmente utilizados, de marcas comerciales conocidas, e incluso, aceptados y aprobados por las organizaciones controladoras (4). De allí la necesidad de que los estudiantes durante su formación profesional adquieran habilidades y destrezas, además deben conocer varios métodos que permitan desarrollar de forma óptima los procedimientos (3).

En la presente investigación, los promedios registrados de cada una de las dimensiones que conformaron el cuestionario sobre conocimiento están muy por debajo de los puntajes ideales en cada una de ellas, según la cantidad de respuestas correctas. Las dimensiones donde se evidenció mayor desconocimiento por parte de los estudiantes de odontología, fueron en las propiedades de los materiales de restauración, seguida de la dimensión 
sobre protocolo clínico para la utilización de cada uno de ellos.

Cada paciente representa una situación de salud única, en condiciones reales, a partir de la cual el estudiante debe lograr la integración y síntesis de conocimiento científico, adquirido previamente, para su resolución integral. Se hace necesario considerar el planteamiento que en el ambiente educativo universitario, los aspectos pedagógicos y científicos constituyen un recurso invalorable para el aprendizaje de contenidos en cualquier área del conocimiento, incluyendo la clínica Integral y los materiales de restauración como tratamiento odontológico. La base fundamental de la práctica clínica individualizada, requiere entrenamiento y conocimiento, que permita la búsqueda de información pertinente (5). Es necesario el entrenamiento de alumnos y docentes en la construcción del saber: aprender a aprender.

En lo correspondiente a la guía de observación para verificar la aplicación de los conocimientos en la práctica clínica, los promedios registrados de cada una de las dimensiones que conformaron el instrumento están igualmente por debajo de los puntajes ideales en cada una de ellas, según la cantidad de aspectos realizados de manera incorrecta o no satisfactoria. Así mismo en los puntajes totales, se tiene que el promedio registrado está muy por debajo de la máxima calificación en la aplicación de los conocimientos sobre materiales restauradores. La dimensión donde se evidenció peor aplicación fue en la selección del material restaurador ideal y el protocolo clínico tanto para la aplicación de resina como para la utilización de vidrio ionomérico. De aquellos estudiantes que evidenciaron un nivel de conocimiento medio sobre materiales de restauración (8 casos), predominaron aquéllos con nivel de aplicación inadecuada (6 casos).

Para Álvarez et al (5) el proceso enseñanza-aprendizaje desarrollado en la clínica requiere de la articulación entre la teoría y la práctica, como dos momentos simultáneos en la construcción del conocimiento. La dificultad de aplicar el fundamento teórico en la práctica no es privativa de la odontología, pues en otras profesiones se ha documentado esta situación $(6,7)$. Estos resultados llaman la atención en tanto se infiere que los objetivos de aprendizaje no se han logrado en su totalidad en los estudiantes objeto de la presente investigación. Conviene mencionar a Spoletti (8) quien sugirió que el aprendizaje en las asignaturas clínicas en odontología implica una realidad distinta a la realidad áulica y pocas veces, abordada en su integralidad desde la percepción de los tres actores del proceso: estudiante-docente-paciente. Si bien este escenario es común a diferentes disciplinas clínicas de las Ciencias de la Salud, adquiere una característica peculiar en la clínica odontológica que es la implementación inmediata del tratamiento planificado, generalmente por parte del mismo estudiante practicante.

Esto hace indispensable aprovechar la relación entre los tres individuos involucrados para propiciar una docencia productiva, cuyo resultado sea beneficioso para la salud del paciente.

Lifshitz (9) ha señalado que el aprendizaje de la clínica apenas puede someterse a las estrategias manejadas en otro tipo de aprendizajes pues, en primer lugar, no se logra mediante memorización y lecturas, las actividades tradicionales de aula son relativamente ineficientes, es muy difícil de sistematizar en la medida en que las oportunidades para la práctica obedecen a la satisfacción de demandas de salud y no a una planificación educativa, 
con lo cual se requiere flexibilidad, integralidad y complementariedad del currículo.

\section{CONCLUSIONES}

$\mathrm{L}$ as dimensiones en las cuales se apreció un mayor porcentaje de desconocimiento por parte de los estudiantes de odontología fueron: propiedades de los materiales de restauración y protocolo clínico para la utilización de cada uno de ellos. Entre el conocimiento sobre los materiales de restauración y su aplicación por parte de los estudiantes existe una mediana relación.

La aplicación en la población estudiada está comprometida tanto en la selección del material restaurador ideal como en el protocolo clínico para la aplicación de biomateriales. Destaca la relación inversa entre el nivel de conocimiento medio sobre materiales de restauración y el nivel de aplicación inadecuada, lo cual sugiere que se hace necesario plantear estrategias curriculares orientadas a trascender la brecha entre teoría y práctica con el fin de alcanzar niveles de excelencia en la práctica profesional del odontólogo para beneficio de la sociedad.

- Conflicto de intereses: Ninguno declarado por el autor.

- Financiación: Autofinanciado.

- Agradecimientos: Ninguno declarado por el autor.

\section{REFERENCIAS}

1. Hervás A, Martínez M, Cabanes J, Barjau A, Fos P. Resinas compuestas: Revisión de los materiales e indicaciones clínicas. Med. Oral patol. oral cir. Bucal [Internet]. 2006 [citado 201814 de febrero]; 11(2): 215220. http://scielo.isciii.es/scielo.php?script=sci_artte xt\&pid=S1698-69462006000200023\&Ing=es.

2. Apaza C, Bustamante G. Propiedades físicas de los biomateriales en odontología. Rev. act. clin. med [Internet]. 2017 [citado 201814 de febrero]. Disponible en: http://www.revistasbolivianas.org.bo/scielo.php ?script=sci_arttext\&pid=S2304$37682013000300001 \& \operatorname{lng}=\mathrm{es}$

3. Restrepo DP, Ardila CM. Reacciones adversas ocasionadas por los biomateriales usados en prostodoncia. Av. Odontoestomatol [Internet]. 2010 [citado 201814 de marzo]; 26(1): 19-30. Disponible

en: http://scielo.isciii.es/scielo.php?script=sci_artte xt\&pid=S0213-12852010000100003\&lng=es

4. López A, Zapata O. Identificación de factores de riesgo durante el uso y manipulación de los materiales dentales y conocimientos de los factores protectivos. En Colombia. [Internet]. $2018 . \quad$ Disponible en: https://encolombia.com/medicinaodontologia/odontologia/uso-y-manipulacionde-los-materiales-dentales/

5. Álvarez L, Gugelmeier V, Hermida L. Cómo aprenden los estudiantes de odontología que cursan el último año de la carrera. Odontoestomatología [Internet]. 2013 mayo [citado 201810 de febrero]; 15(21): 4-11. Disponible en: http://www.scielo.edu.uy/scielo.php?script=sci arttext\&pid=S1688$93392013000100002 \& \operatorname{lng}=\mathrm{es}$

6. Sancho D. Teorías y modelos en la práctica enfermera: ¿Un binomio imposible? Enfermo. Glob. [Internet]. 2012 [citado 201710 de marzo]; 11(27): 292298.doi.org/10.4321/S1695-

61412012000300015

7. León C. Los problemas profesionales generales de enfermería en el diseño curricular. Rev. cubana enfermer [Internet]. 2005 Abr [citado 201816 de enero]; 21(1): 1-1. Disponible en: http://scielo.sld.cu/scielo.php?script=sci_arttext \&pid=S0864-03192005000100008\&lng=es

8. Spoletti P. La enseñanza en la clínica odontológica. Rev educ cienc salud [Internet]. 2014 [citado 201814 de marzo]; 11 (2): 166$170 . \quad$ Disponible en:http://www2.udec.cl/ofem/recs/anteriores/vo I1122014/artrev11214a.htm

9. Lifshitz A. La enseñanza de la competencia clínica. Gac. Méd. Méx [Internet]. 2004 [citado 2018 febrero 14]; 140(3): 312-313. Disponible en:

http://www.scielo.org.mx/scielo.php?script=sci arttext\&pid=S0016$38132004000300010 \&$ Ing =es. 